Pacific Journal of Mathematics

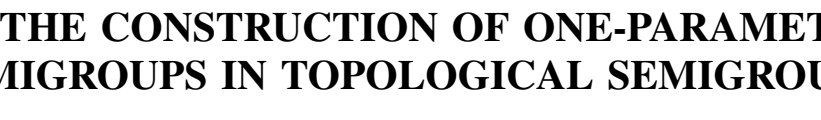




\title{
ON THE CONSTRUCTION OF ONE-PARAMETER SEMIGROUPS IN TOPOLOGICAL SEMIGROUPS
}

\author{
JOHN YUAN
}

\begin{abstract}
Let $S$ be a topological Hausdorff semigroup and $s \in S$ be a strongly root compact element. Then there are an algebraic morphism $f: Q_{+} \cup\{0\} \rightarrow S$ with $f(0)=e, f(1)=s$, and a oneparameter semigroup $\phi: H \rightarrow S$ which satisfy the following properties: If $K=\bigcap\{f(] 0, \varepsilon[Q): 0<\varepsilon<1\}$, then $K$ is a compact connected abelian subgroup of $\mathscr{H}(e), \phi(0)=e, \phi(H)$ is in the centralizer $Z=\{x \in e S e: x k=k x$ for all $k \in K\}$ of $K$ in $e S e$, and $\phi(t) \in f(t) K$ for each $t \in Q_{+}$. Furthermore, if $\mathscr{U}$ is any neighborhood of $s$ in $S$, then $\phi$ may be chosen so that $\phi(1) \in \mathscr{U}$ : and, in fact, if $K$ is arcwise connected, then $\phi$ may be chosen so that $\phi(1)=s$. The above statements also hold for strongly $p$ th root compact elements almost everywhere.
\end{abstract}

1. Introduction. We are concerned with the question of when a divisible element in a topological semigroup can be embedded in a one-parameter semigroup which has many applications in Probability theory (cf. [4], [8]).

The first result about the existence of one-parameter semigroups in a compact semigroup which we call the One-Parameter Semigroup Theorem is due to Mostert and Shields [7], 1957. In 1960, an independent proof based on the local nature of the compact semigroup was given by Hoffmann (cf. [5], [6]). In 1970, a global proof was presented by Carruth and Lawson [1]. The first result of a generalized one-parameter semigroup theorem dealing with the embedding problems which we will call the Embedding and Density Theorem is indicated by Hofmann in [4] and later proved by Siebert [8]. Siebert's proof is based on the notion of a local semigroup called ducleus (cf. [6]). We will present in this paper a global proof of this theorem by applying the One-Parameter Semigroup Theorem.

Throughout this paper, we maintain that $R_{+}, Q_{+}$and $Z_{+}$are the totalities of strictly positive real numbers, rational numbers and integers, respectively, $\boldsymbol{H}=R_{+} \cup\{0\}$ and $Q_{+}^{p}=\left\{n / p^{m}: n \in Z_{+}\right.$, $\left.m \in Z_{+} \cup\{0\}\right\}$ for a prime $p$. For convenience, we will use $\left.] a, b\right]_{\ell}$ (resp. $] a, b\left[{ }_{Q}\right.$, etc.) and $\left.] a, b\right]_{Q^{p}}($ resp. $] a, b\left[Q_{Q^{p}}\right)$ to denote $\left.] a, b\right] \cap Q_{+}$ (resp. $] a, b\left[\cap Q_{+}\right.$, etc.) and $\left.] a, b\right] \cap Q_{+}^{p}$ (resp. $] a, b\left[\cap Q_{+}^{p}\right.$ ) respectively. We also maintain that $S$ is a topological (Hausdorff) semigroup and $\mathscr{H}(e)$ is the maximal group of units in the closed subsemigroup eSe for an idempotent $e \in S$. 
2. On the existence of a one-parameter semigroup in $\overline{f(A)}$ where $f: A \rightarrow S$ is an algebraic morphism with $A=Q_{+}, Q_{+}^{p}$. Throughout this section, we will always assume that $f: Q_{+}$(resp. $\left.Q_{+}^{p}\right) \rightarrow S$ is an algebraic morphism so that $\overline{\left.f(10, d]_{Q}\right)}$ (resp. $\overline{\left.f(] 0, d]_{Q^{p}}\right)}$ ) is compact for some $d>0$ unless mentioned otherwise. As the discussions for $Q_{+}$and for $Q_{+}^{p}$ would be almost the same, we will concentrate on $Q_{+}$only.

Definition. For each $s \in S$ and each $n \geqq 1$, let $W_{n}(s)=\{t \in S$ : $\left.t^{n}=s\right\}, W(n ; s)=\left\{t^{m}: 1 \leqq m \leqq n, t^{n}=s\right\} . \quad s$ is said to be divisible (resp. $p$-divisible) if $W_{n}(s) \neq \varnothing\left(\right.$ resp. $\left.W_{p^{n}}(s) \neq \varnothing\right)$ for all $n \geqq 1$; root compact (resp. pth root compact) if $W_{n}(s)$ (resp. $W_{p^{n}}(s)$ ) is in addition compact for each $n \geqq 1$; strongly root compact (resp. strongly $p$ th root compact) if $W_{\infty}(s)=U\{W(n ; s): n \geqq 1\} \quad$ (resp. $W_{p^{\infty}}(s)=U\left\{W\left(p^{n} ; s\right)\right.$ : $n \geqq 1\})$ is in addition relatively compact.

Proposition 2.1. Let $s$ be a root compact (resp. $p$ th root compact) element in $S$. Then there is an algebraic morphism $f: Q_{+}$(resp. $\left.Q_{+}^{p}\right) \rightarrow S$ so that $f(1)=s$. If $s$ is strongly root compact (resp. strongly $p$ th root compact), then $f$ may be chosen so that $\overline{\left.f(10,1]_{Q}\right)}$ (resp. $\overline{\left.f(10,1]_{Q^{p}}\right)}$ ) is compact.

Proof. For each $n \geqq 1$ and $i \geqq 0$, pick an $s_{n+i} \in W_{(n+i) !}(s)$ (resp. $\left.s_{n+\imath} \in W_{p^{(n+i)}}(s)\right)$ and let

$$
\begin{gathered}
a_{n}=\left(s_{n}^{n !}, s_{n}^{n ! / 2 !}, \cdots, s_{n}, s_{n+1}, \cdots\right) \\
\left(\text { resp. } a_{n}=\left(s_{n}^{p^{n}}, s^{p^{n-1}}, \cdots, s_{n}, s_{n+1}, \cdots\right)\right) .
\end{gathered}
$$

Then $\left\{a_{n}\right\}$ is a sequence in the compact set $\Pi_{n \geqq 1} W_{n !}(s)$ (resp. $\left.\prod_{n \geqq 1} W_{p^{n}}(s)\right)$. Hence there is a convergent subnet $\left\{a_{n(k)}\right\}$ converging to $a=\left(t_{1}, t_{2}, \cdots\right) \in \Pi_{n \geqq 1} W_{n !}(s)$ (resp. $\left.\prod_{n \geqq 1} W_{p^{n}}(s)\right)$.

Then

$$
\begin{aligned}
t_{q+1}^{q+1}= & \left(\lim s_{n(k)}^{n(k) ! /(q+1) !}\right)^{q+1} \\
= & \lim s_{n(k)}^{n(k) ! / q !}=t_{q} \\
\text { (resp. } t_{q+1}^{p} & =\left(\lim s_{n(k)}^{p^{n(k)-q}}\right)^{p} \\
& \left.=\lim s_{n(k)}^{p^{n(k)-q+1}}=t_{q}\right)
\end{aligned}
$$

for all $q \geqq 1$, and $t_{1}=s$. If $n / m !=b / a !\left(\operatorname{resp} . n / p^{m}=b / p^{a}\right)$, then

$$
\begin{gathered}
t_{m}^{n}=\left(t_{m}^{m ! / a !}\right)^{b}=t_{b}^{a} \\
\text { (resp. } \left.t_{m}^{n}=\left(t_{m}^{p^{m-a}}\right)^{b}=t_{a}^{b}\right) .
\end{gathered}
$$

Hence $f: Q_{+}\left(\operatorname{resp} . Q_{+}^{p}\right) \rightarrow S$ given by $f(n / m !)=t_{m}^{n}\left(\operatorname{resp} . f\left(n / p^{m}\right)=t_{m}^{n}\right)$ 
is well-defined. If $n / m !, b / a ! \in Q_{+}$(resp. $n / p^{m}, b / p^{a} \in Q_{+}^{p}$ ), assuming $a \geqq m$, then

$$
\begin{aligned}
\begin{aligned}
f(n / m !+b / a !) & =f\left(\frac{n(a ! / m !)+b}{a !}\right) \\
& =t_{a}^{n(a ! / m !)} t_{a}^{b}=t_{m}^{n} t_{a}^{b}
\end{aligned} \\
\text { resp. } f\left(n / p^{m}+b / p^{a}\right)=f\left(\frac{n p^{a-m}+b}{p^{a}}\right) \\
\left.=t_{a}^{n p^{a-m}} t_{a}^{b}=t_{m}^{n} t_{a}^{b}\right),
\end{aligned}
$$

whence $f$ is an algebraic morphism so that $f(1)=s$. The rest is simple.

Lemma 2.2. for each $x>0$, let $S(x)=\overline{f(10, x[Q)}$. Then

(1) $S(x+y)=S(x) S(y)$ for all $x, y>0$. In particular, $S(x)$ is compact for each $x>0$

(2) $\overline{f\left(Q_{+}\right)}$has the identity e so that $K=\cap\left\{S(x): x \in Q_{+}\right\}$is a divisible compact abelian subgroup of $\mathscr{H}(e)$. In particular, we may extend $f$ to $Q_{+} \cup\{0\}$ so that $f(0)=e$

(3) $\overline{K f\left(\left[x, y\left[_{Q}\right)\right.\right.}=\overline{f\left(\left[x, y\left[_{Q}\right)\right.\right.}$ for all $x<y \in Q_{+}$.

Proof. Straightforward (cf. §3, Chapter B, [6]).

LEMMA 2.3. The following statements are equivalent:

(1) $K=\{f(0)\}$

(2) $f$ is continuous at 0

(3) $f$ is continuous.

Proof. (cf. 3.9, p. 102, [6].)

LEMma 2.4. If $f$ is continuous, then there is a unique oneparameter semigroup $\phi$ so that $\phi \mid\left(Q_{+} \cup\{0\}\right)=f$.

Proof. Given a $d>0$, there is a net $\left\{x_{\alpha}\right\}$ in $] 0, d+1\left[\left[_{Q}\right.\right.$ with $\lim x_{\alpha}=d$. Since $\left\{\left(f\left(x_{\alpha}\right)\right\}\right.$ is a net in $S(d+1)$, there is a convergent subnet $\left.\left\{f x_{\beta}\right)\right\}$. Define $F(d)=\lim f\left(x_{\beta}\right)$. It is straightforward to check that $F: H \rightarrow S$ is a well defined morphism so that $\cup \overline{\{F(] 0, x[) \text { : }}$ $x>0\}=\{f(0)\}$, whence $F$ is continuous (cf. 3.9, p. 102, [6]).

Lemma 2.5. Let $\phi: H \rightarrow S$ be a nontrivial one-parameter semigroup. Then there is a $d \in] 0,1]$ so that $\phi \mid[0, d]$ is injective. Moreover, if $c>0$, one may reparameterize $\phi$ so that $\phi \mid[0, c]$ is injective (cf. 3.9, p. 102, [6]). 
Since $K$ acts on $\overline{f\left(Q_{+}\right)}$and $\overline{f([x, y[Q)}$, one has the orbit spaces $\overline{f\left(Q_{+}\right) / K}$ and $\overline{f([v, y[Q) / K}$. We will use the same letter $\pi$ to denote the orbit maps.

LEMMA 2.6. $\overline{f\left(Q_{+}\right)} / K$ is a topological monoid under the multiplication $x K \cdot y K=x y K$.

Lemma 2.7. If $f\left(Q_{+}\right) \not \subset K$, then $\pi \circ f: Q_{+} \cup\{0\} \rightarrow \overline{f\left(Q_{+}\right)} / K$ is nontrivial continuous morphism so that $\pi\left(\overline{f\left(\left[x, y\left[_{Q}\right)\right.\right.}\right)=\overline{f([x, y[Q)} / K$ for all $x<y \in Q_{+} \cup\{0\}$.

Proof. The continuity of $\pi \circ f$ follows from 2.3. The rest follows from the closedness of $\pi$.

In the remainder of this section, we maintain that $f(1) \notin K$ and so $\pi \circ f$ extends to a unique one-parameter semigroup $g: \boldsymbol{H} \rightarrow \overline{f\left(Q_{+}\right)} / K$ that $g \mid[0,2]$ is injective by a suitable reparameterization of $g$ or $f$, i.e. the following diagram commutes:

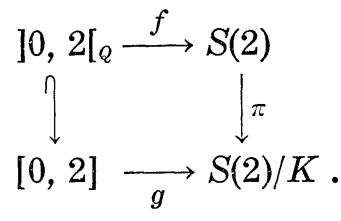

Let $\rho=g^{-1} \circ \pi: S(2) \rightarrow[0,2]$. Then $\rho$ is a continuous map such that

$$
\rho(f(r))=\left(g^{-1} \circ \pi\right)(f(r))=r \text { for all } r \in[0,2]_{Q}
$$

and that the following condition is satisfies:

$$
\rho(x y)=\rho(x)+\rho(y) \text { for all } x, y \in S(1) .
$$

LEMMA 2.8. The following statements hold:

(1) $x \in K f(r)$ iff $x \in \pi^{-1}(g(r))$ for each $r \in Q_{+} \cup\{0\}$

(2) $x \in S(2)$ iff there is a unique $t \in[0,2]$ so that $x \in \pi^{-1}(g(t))$

(3) $\pi^{-1}(g([x, y]))=\overline{K \overline{f([x, y[Q)}}=\overline{f(] x, y[Q)}$ for all $x, y \in Q_{+} \cup\{0\}$

(4) $S(1) K f(1) \subset K \overline{f([1,2[Q)}$

(5) $S(1) \backslash K f(1)=S(2) \backslash K \overline{f([1,2[Q)}$.

Proof. Straightforward.

Define a multiplication on the space $X$ obtained from $S(1)$ by collapsing $K f(1)$ to a point as follows:

$$
m_{R}(x, y)= \begin{cases}x y & \text { if } \quad x, y, x y \in S(1) \backslash K f(1) \\ K f(1) & \text { otherwise. }\end{cases}
$$


Let $\pi^{\prime}: S(2) \rightarrow X$ be defined via

$$
\begin{aligned}
& \pi^{\prime}|S(1) \backslash K f(1)=\pi| S(2) \backslash K \overline{f\left(\left[1,2\left[{ }_{Q}\right)\right.\right.} \text { and } \\
& \left.\pi^{\prime}(K \overline{f([1,2[Q})\right)=\{K f(1)\}
\end{aligned}
$$

then

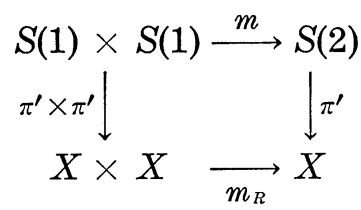

commutes, hence $m_{R}$ is a global multiplication on $X$.

Lemma 2.9. $X$ is a compact abelian monoid in the quotient topology.

Proof. Since $\pi^{\prime}$ is a closed map, $m_{R}$ is continuous.

Let $[0,1]_{*}$ denote the space $[0,1]$ equipped with the multiplication $x+y=\min \{1, x+y\}$. Then $[0,1]_{*}$ is a compact monoid in the usual topology. In particular, we have the following factorization:

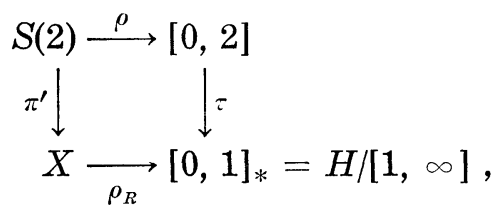

where $\tau: H \rightarrow[0,1]_{*}$ is the canonical map and $\rho_{R}: X \rightarrow[0,1]_{*}$ is the unique continuous morphism making the diagram commute.

LEMma 2.10. The following statements hold:

(1) $X$ has exactly two idempotents e and $0 \equiv K f(1)$

(2) $K$ is the maximal group of units in $X$

(3) $K$ is not open in $X$

(4) $X \backslash\{0\}$ is isomorphic to $S(1) \backslash K f(1)$.

Proof. (1) and (4) are clear. (2): We have $\left.\left.X \backslash K=\rho_{R}^{-1}(] 0,1\right]\right)$ which is an ideal. Thus $K$ is maximal. (3): If $K$ were open, then $X \backslash K$ would be closed, hence compact, and thus $\left.\rho_{R}(X \backslash K)=10,1\right]$ would be compact which is not the case.

Proposition 2.11. There is a continuous morphism $\phi_{*}:[0,1]_{*} \rightarrow X$ so that $\phi_{*}(0)=e$ and $\phi_{*}^{-1}(\{0\})=\{1\}$. 
Proof. By 2.10 we can apply the One-Parameter Semigroup Theorem (Thm. 1, p. 510, [7]; [1]) to obtain $\phi_{*}$.

Proposition 2.12. $\rho_{R^{\circ}} \phi_{*}$ is the identity $\operatorname{map}$ on $[0,1]_{*}$.

Proof. We observe first that $\rho_{R^{\circ}} \phi_{*}$ is an endomorphism $\alpha$ of $[0,1]_{*}$ with $\alpha^{-1}(\{1\})=\{1\}$ and is therefore the identity.

Proposition 2.13. There is a one-parameter semigroup $\phi: H \rightarrow S$ such that $\phi(r) \in K f(r)$ for all $r \in Q_{+}$.

Proof. For all $r \in\left[0,1\left[_{Q}, r=\rho_{R^{\circ}} \phi_{*}(r)=\rho \circ \phi_{*}(r)\right.\right.$ and so $\phi_{*}(r) \in$ $\rho^{-1}(r)=K f(r)$. Let $\phi$ be the unique lifting of $\phi_{*}$ to $\boldsymbol{H}$. Then $\phi(r) \in$ $K f(r)$ for all $r \in Q_{+}$.

\section{On the Embedding and Density Theorem.}

Proposition 3.1. Let $G$ be a locally compact abelian group and $L G=\operatorname{Hom}(R, G)$ the totality of one-parameter subgroups in $G$. If exp: $L G \rightarrow G$ denotes the map $\exp (f)=f(1)$, then

(1) $\overline{\exp (G L)}=G_{0}$, where $G_{0}$ is the identity component of $G$

(2) $\exp (L G)=G_{0}$ iff $G_{0}$ is arcwise connected.

Proof. (1) (25.20, p. 410, [3]). (2) (Thm. 1, p. 40, [2]).

Embedding AND Density Theorem 3.2. Let $s$ be strongly root compact in $S$. Then there are an algebraic morphism $f: Q_{+} \cup\{0\} \rightarrow S$ with $f(0)=e, f(1)=s$, and a one-parameter semigroup $\phi: H-S$ which satisfy the following properties: If $K=\cap\{\overline{f(] 0, \varepsilon[Q)}: 0<\varepsilon<1\}$, then $K$ is a compact connected abelian subgroup of $\mathscr{H}(e), \phi(0)=e$, $\phi(\boldsymbol{H})$ is in the centralizer $Z=\{x \in e S e: x k=k x$ for all $k \in K\}$ of $K$ in eSe, and $\phi(t) \in K f(t)$ for each $t \in Q_{+}$.

Furthermore, if $\mathscr{U}$ is any neighborhood of $s$ in $S$, then $\phi$ may be chosen so that $\phi(1) \in \mathscr{L}$; and, in fact, if $K$ is arcwise connected, then $\phi$ may be chosen so that $\phi(1)=s$.

Proof. By 2.1, there is an algebraic morphism $f: Q_{+} \cup\{0\} \rightarrow S$ such that $f(0)=e, f(1)=s, \overline{\left.f(10,1]_{\ell}\right)}$ is compact, $K \subset \mathscr{H}(e)$ is a compact connected abelian subgroup and $\overline{f\left(Q_{+}\right)} \subset e S e$.

If $s \in K$, then by 3.1 the assertion is true. If $s \notin K$, then by 2.13 there is a one-parameter semigroup $\phi: H \rightarrow S$ so that $\phi(\boldsymbol{H}) \subset$ $\overline{f\left(Q_{+}\right)} \subset e S e$ and $\phi(r) \in K f(r)$ for all $r \in Q_{+} \cup\{0\}$. In particular, $\phi(\boldsymbol{H})$ is in the centralizer of $K$ in $e S e$. Let $\mathscr{Q}$ be a neighborhood of $s$ in $S$; then there is a neighborhood $U$ of $e$ in $K$ so that $s U \subset \mathscr{U}$. Pick 
a $k \in K$ so that $\phi(1)=s k$, by the fact that $\overline{\exp (L K)}=K$, there is an $\psi \in L K$ so that $\psi(1) \in U k^{-1}$. Let $\phi_{1}: H \rightarrow S$ be defined via $\phi_{1}(r)=$ $\phi(r) \psi(r)$. As $\phi(\boldsymbol{H})$ is in the centralizer of $K$ in $e S e$, then $\phi_{1}$ is a well-defined one-parameter semigroup so that

$$
\phi_{1}(1)=\phi(1) \psi(1) \in \operatorname{sk} U k^{-1}=s U .
$$

It is easy to check that $\phi_{1}$ also satisfies the same properties as stated above. If $K$ is arcwise connected, by $3.1 \psi$ may be chosen so that $\psi(1)=k^{-1}$ and so $\phi_{1}(1)=s$.

CoROLlaRY 3.3. If $K$ is a Lie group, then there is a oneparameter semigroup $\phi$ so that $\phi(1)=s$ (cf. Thm. 7, p. 141, [9]).

THEOREM 3.4. Let $s$ be a strongly pth root compact element in $S$. Then there are an algebraic morphism $f: Q_{+}^{p} \cup\{0\} \rightarrow S$ with $f(0)=e$, $f(1)=s$, and a one-parameter semigroup $\phi: H \rightarrow S$ which satisfy the following properties: If $K_{p}=\cap\left\{\overline{f(] 0, \varepsilon\left[Q_{Q^{p}}\right)}: 0<\varepsilon<1\right\}$, then $K_{p}$ is a p-divisible compact abelian subgroup of $\mathscr{H}(e), \phi(0)=e, \phi(\boldsymbol{H})$ is in the centralizer $Z$ of $K_{p}$ in eSe, and $\phi(r) \in K_{p} f(r)$ for all $r \in Q_{+}^{p}$.

REMARK. $K_{p}$ is in general not divisible (cf. p. 265, [5]; p. 117, [6]).

Proposition 3.5. Let $s$ be a strongly root compact (resp. strongly $p$ th root compact) element in $S$ and $f$ and $\phi$ be as stated in 3.2 (resp. 3.4). Then there is an algebraic morphic morphism $h: Q_{+} \rightarrow K$ (resp. $h: Q_{+}^{p} \rightarrow K_{p}$ ) so that $\dot{\phi}(r)=f(r) h(r)$ for all $r \in Q_{+}$(resp. $Q_{+}^{p}$ ).

Proof. For each $n \geqq 1$, let $A_{n !}=\{x \in K: f(1 / n !) x=\phi(1 / n !)\}$ (resp. $\left.B(p ; n)=\left\{x \in K_{p}: f\left(1 / p^{n}\right) x=\phi\left(1 / p^{n}\right)\right\}\right)$. Clearly, $A_{n !}(\operatorname{resp} . B(p ; n)$ is a nonempty compact subset for each $n \geqq 1$. The construction of $h$ then follows as in 2.1.

The following example shows that there are elements which are not strongly root compact but which are neverthless embeddable in one-parameter semigroups:

EXAmple 3.5. Let $S=S L(2 ; R)$ and $s=\left(\begin{array}{rr}-1 & 0 \\ 0 & -1\end{array}\right)$ : then $s$ is divisible and $W_{2}(s) \supset\left\{\left(\begin{array}{ll}0 & y \\ z & 0\end{array}\right): y z=-1\right\}$ is not compact, whence $s$ is not even 2 th root compact. But the map $f: R \rightarrow S$ defined via

$$
f(t)=\left(\begin{array}{r}
\cos \pi t \sin \pi t \\
-\sin \pi t \cos \pi t
\end{array}\right)
$$


is a one-parameter subgroup so that $f(1)=s$.

ACKNOWLeDGMents. The author wishes to thank Drs. Karl $\mathrm{H}$. Hofmann, Michael W. Mislove and John R. Liukkonen for many helpful suggestions.

\section{REFERENCES}

1. J.H. Carruth and J.D. Lawson, On the existence of one-parameter semigroups, Semigroup Forum, 1 (1970), 85-90.

2. J. Dixmier, Quelques propriétés des groupes abélian localement compacts, Bull. Sci. Math. $2^{\text {e }}$ série, 85 (1957), 38-48.

3. E. Hewitt and K. A. Ross, Abstract Harmonic Analysis I, Springer-Verlag, BerlinHeidelberg-New York, (1963/70).

4. H. Heyer, Infinitely divisible probability measures on compact groups, Lecture Notes in Mathematics 247, Springer-Verlag, Heidelberg (1973), 55-247.

5. K. H. Hofmann, Topologisches Halbgruppen mit dichter submonogener Unterhalbgruppe, Math. Zeitschrift, 74 (1960), 232-276.

6. K. H. Hofmann and P.S. Mostert, Elements of Compact Semigroups, Charless E. Merrill, Columbus, Ohio (1966).

7. P. S. Mostert and A. L. Shields, One-parameter semigroups in a semigroups, Trans. Amer. Math. Soc., 96 (1960), 510-517.

8. E. Seibert, Einbettung unendlich teilbarer Wahrscheinlichkeitsmasse auf topologischen Gruppen, Z. Wahrscheinlichkeitstheorie verw. Gebiete, 28 (1974), 227-247.

9. Ph. Tondeur, Introduction to Lie Groups and Transformation Groups, Second Edition, Springer-Verlag. Berlin (1969).

Received November 13, 1975 and in revised form February 9, 1976.

National Tsing Hua University, Taiwan 300 


\section{PACIFIC JOURNAL OF MATHEMATICS}

\section{EDITORS}

RICHARD ARENS (Managing Editor) University of California

Los Angeles, California 90024

R. A. Beaumont

University of Washington

Seattle, Washington 98105
J. DUGUNDJI

Department of Mathematics University of Southern Californı

Los Angeles, California 90007

D. Gilbarg and J. Milgram

Stanford University

Stanford, California 94305

\section{ASSOCIATE EDITORS}
E. F. BECKENBACH
B. H. NeumanN
F. WOLF
K. YosHIDA

\section{SUPPORTING INSTITUTIONS}

UNIVERSITY OF BRITISH COLUMBIA

CALIFORNIA INSTITUTE OF TECHNOLOGY

UNIVERSITY OF CALIFORNIA

MONTANA STATE UNIVERSITY

UNIVERSITY OF NEVADA

NEW MEXICO STATE UNIVERSITY

OREGON STATE UNIVERSITY

UNIVERSITY OF OREGON

OSAKA UNIVERSITY
UNIVERSITY OF SOUTHERN CALIFORNIA

STANFORD UNIVERSITY

UNIVERSITY OF TOKYO

UNIVERSITY OF UTAH

WASHINGTON STATE UNIVERSITY

UNIVERSITY OF WASHINGTON

* * * *

AMERICAN MATHEMATICAL SOCIETY

NAVAL WEAPONS CENTER 


\section{Pacific Journal of Mathematics \\ Vol. 65, No. $1 \quad$ September, 1976}

David Lee Armacost, Compactly cogenerated LCA groups ............. 1

Sun Man Chang, On continuous image averaging of probability measures ...... 13

J. Chidambaraswamy, Generalized Dedekind $\psi$-functions with respect to a

polynomial. II................................... 19

Freddy Delbaen, The Dunford-Pettis property for certain uniform algebras ..... 29

Robert Benjamin Feinberg, Faithful distributive modules over incidence

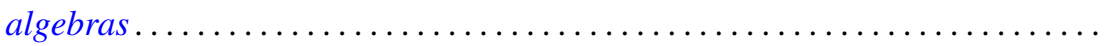

Paul Froeschl, Chained rings . . . . . . . . . . . . . . . . . . . .

John Brady Garnett and Anthony G. O'Farrell, Sobolev approximation by a sum

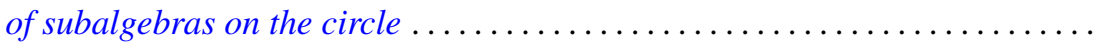

Hugh M. Hilden, José M. Montesinos and Thomas Lusk Thickstun, Closed

oriented 3-manifolds as 3-fold branched coverings of $S^{3}$ of special type.....

Atsushi Inoue, On a class of unbounded operator algebras ................

Peter Kleinschmidt, On facets with non-arbitrary shapes.

Narendrakumar Ramanlal Ladhawala, Absolute summability of Walsh-Fourier

series

Howard Wilson Lambert, Links which are unknottable by maps . . . . . . . . . . .

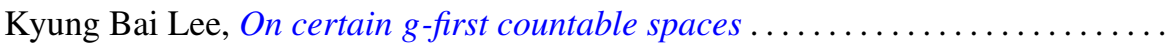

Richard Ira Loebl, A Hahn decomposition for linear maps .................

Moshe Marcus and Victor Julius Mizel, A characterization of functionals on $W_{1}^{p}$ possessing autonomous kernels. I . .

James Miller, Subordinating factor sequences and convex functions of several

variables.

Keith Pierce, Amalgamated sums of abelian l-groups ...

Jonathan Rosenberg, The $C^{*}$-algebras of some real and $p$-adic solvable

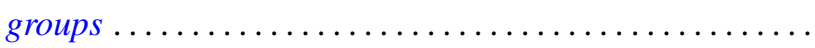

Hugo Rossi and Michele Vergne, Group representations on Hilbert spaces defined

in terms of $\partial_{b}$-cohomology on the Silov boundary of a Siegel domain . .

Mary Elizabeth Schaps, Nonsingular deformations of a determinantal

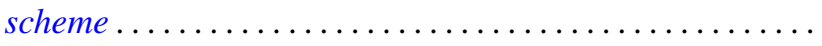

S. R. Singh, Some convergence properties of the Bubnov-Galerkin method...

Peggy Strait, Level crossing probabilities for a multi-parameter Brownian

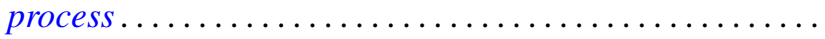

Robert M. Tardiff, Topologies for probabilistic metric spaces .

Benjamin Baxter Wells, Jr., Rearrangements of functions on the ring of integers of

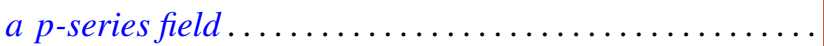

Robert Francis Wheeler, Well-behaved and totally bounded approximate identities for $C_{0}(X)$.

Delores Arletta Williams, Gauss sums and integral quadratic forms over local

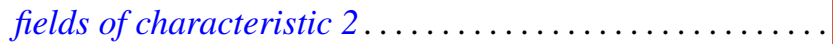

John Yuan, On the construction of one-parameter semigroups in topological 